\title{
THE EXIT CHARACTERISTICS OF MARKOV PROCESSES WITH APPLICATIONS TO CONTINUOUS MARTINGALES IN $\boldsymbol{R}^{n}$
}

\author{
BY \\ GENE DENZEL
}

Introduction. The main results of this paper were announced in [4]. A weaker form of the results in $\$ \S 1$ and 2 appeared in the author's thesis $\left({ }^{1}\right)$ [5], the research for which was supported by the National Science Foundation.

Paul Levy showed in [11] that Brownian motion on the line can be characterized in terms of two martingale functions. Recently Arbib [1] extended this result to include all diffusions on the line with natural boundaries at infinity. His method was to characterize a diffusion by its "exit characteristics", but his proof was only of use in the specific cases he was considering. In $\S 1$ we obtain, by probabilistic methods, a similar characterization of minimal (no return from the boundary) right continuous strong Markov processes on a general state space. In $\$ 2$ we use this to generalize Levy's theorem to $\boldsymbol{R}^{n}$, and in $\$ 4$ indicate some extension of Arbib's results to $\boldsymbol{R}^{n}$. In $\S 3$ we make use of our general Levy-type theorem to obtain a characterization of all continuous martingales in $\boldsymbol{R}^{n}$, extending a result of Dambis [3] on the line. It was recently brought to my attention that Kunita and Watanabe [10] have used quite different methods to obtain somewhat weaker results which were also, in essence, reported by Dubins and Schwarz [7], who used still another method of proof.

0. Preliminaries. We collect in this section some definitions and facts which will be needed in the sequel. By a stochastic process $X=\left(X_{t}, \mathscr{F}_{t}\right)$ we shall mean a family of random variables $X_{t}(t \in[0, \infty[)$ on a probability space $(\Omega, \mathscr{F}, \boldsymbol{P})$, with values in a space $(E, \mathscr{B})$, and an increasing family of sub- $\sigma$-fields $\mathscr{F}_{t} \subset \mathscr{F}$, such that $X_{t}$ is $\mathscr{F}_{t}$-measurable. For our purposes we take $E$ to be a locally compact (Hausdorff) space with a countable base, or the one-point compactification $\bar{E}=E \cup\{\Delta\}$ of such a space, where $\Delta$ is taken to be an isolated point if $E$ is compact. In either case we equip the space with its Borel $\sigma$-field $\mathscr{B}$, or $\overline{\mathscr{B}}$. We deal only with processes whose paths are right continuous for $t \in[0, \infty[$ and have left limits for $t \in] 0, \infty$ [. It will be convenient to define $X_{\infty}=\lim _{t \rightarrow \infty} X_{t}$ if the limit exists in the topology of $\bar{E}$, and $X_{\infty}=\Delta$ otherwise. The point $\Delta$ is assumed to be a trap, i.e., if

Presented to the Society, October 30, 1965 under the title A charact ization of Markov processes in terms of their hitting characteristics; received by the editors Oct ber 24, 1966.

( ${ }^{1}$ I wish to take this opportunity to express my appreciation to Professor R. M. Blumenthal, whose helpful suggestions and constructive criticism made the thesis much more substantial than it would have been. 
$X_{t}(w)=\Delta$, then $X_{t+s}(w)=\Delta$ for all $s \geqq 0$. We will feel free to enlarge $\Omega$ as much as is necessary, and will for instance assume that it is closed under the shift transformation $\theta_{t}, X_{s}\left(\theta_{t} w\right)=X_{s+t}(w)$. For much of our work it will be convenient to use the path space representation of a process (see Meyer [12, Chapter IV]).

A stopping time (Markov time) $T$ of an increasing family of $\sigma$-fields $\left(\mathscr{F}_{t}\right)\left(\mathscr{F}_{t} \subset \mathscr{F}\right)$ is a function $T: \Omega \rightarrow[0, \infty]$ such that $\{T \leqq t\} \in \mathscr{F}_{t}$ for $t \geqq 0$. The shift $\theta_{T}$ is defined by $X_{s}\left(\theta_{T} w\right)=X_{s+T(w)}(w)$. The $\sigma$-field of "events prior to $T$ " consists of all $A \in \mathscr{F}$ such that $A \cap\{T \leqq t\} \in \mathscr{F}_{t}$ for $t \geqq 0$. In the sequel we will assume, with no loss in generality, that the collection $\left(\mathscr{F}_{t}\right)$ is right continuous, i.e., that

$$
\mathscr{F}_{t}^{+}=\bigcap_{s>t} \mathscr{F}_{s}=\mathscr{F}_{t}
$$

for $t \geqq 0$. In this case a useful example of a stopping time is $T_{G}=\inf \left\{t>0: X_{t} \in G\right\}$, when $G$ is open (we will take the infimum of an empty set of real numbers to be $+\infty)$. The lifetime $\sigma$ of a process $X$ is defined to be $\sigma=\inf \left\{t>0: X_{t}=\Delta\right\}$, and is easily seen to be also a stopping time.

A real-valued process $X=\left(X_{t}, \mathscr{F}_{t}\right)$ is a martingale (super-martingale, submartingale) if $\boldsymbol{E}\left[\left|X_{t}\right|\right]<\infty$ and $\boldsymbol{E}\left[X_{t+s} \mid \mathscr{F}_{t}\right]=X_{t}$ (respectively $\leqq$, $\geqq$ ) for all $t, s \geqq 0\left(\boldsymbol{E}[\cdot]\right.$ denotes the expectation (integral) with respect to $\boldsymbol{P}$, and $\boldsymbol{E}\left[\cdot \mid \mathscr{F}_{t}\right]$ the conditional expectation with respect to the $\sigma$-field $\mathscr{F}_{t}$ ). A process is called a local martingale, etc., if there is a sequence $\left(T_{n}\right)$ of a.s. finite stopping times increasing to $\infty$ a.s., such that for each $n$ the process stopped at $T_{n}$ (the process $\tilde{X}$ with paths $\tilde{X}_{t}=X_{t \wedge T_{n}}$ ) is a martingale, etc. For details on the general theory of processes, and particularly on martingales and stopping times, the reader is referred to Meyer [12]. The main result we need is the following, the proof of which is a routine use of the results in Chapter VI of Meyer.

THEOREM 0.1. If $X$ is a continuous, positive, local submartingale, then $X$ has a (Meyer) decomposition $X=M\{X\}+A\{X\}$, where $A=A\{X\}$ is a continuous increasing process equal to 0 at $t=0$, and $M$ is a continuous local martingale.

A process $X$ is called a Markov process if $\boldsymbol{E}\left[I_{B} \circ X_{t} \circ \theta_{s} \mid \mathscr{F}_{s}\right]=\boldsymbol{E}\left[I_{B} \circ X_{t} \circ \theta_{s} \mid X_{s}\right]$ a.s. for all $B \in \mathscr{B}$ and $s, t \geqq 0$. It is called a strong Markov process if $s$ can be replaced by any stopping time $S$.

We will call an increasing family of stopping times $\left(\tau_{t}\right)$ for the family $\left(\mathscr{F}_{t}\right)$ a random time change, and write $T=\left(\mathscr{F}_{t}, \tau_{t}\right)$. Corresponding to each increasing process $A=\left(A_{t}, \mathscr{F}_{t}\right)$ there is a random time change $T=\left(\mathscr{F}_{t}, \tau_{t}\right)$, where

$$
\tau_{t}=\inf \left\{u: A_{u}>t\right\} .
$$

We write $T=A^{-1}$ in this case. Given a process $X=\left(X_{t}, \mathscr{F}_{t}\right)$, a random time change $T=\left(\mathscr{F}_{t}, \tau_{t}\right)$ is called $X$-continuous if the function $t \rightarrow X_{t}$ is a.s. constant on all intervals $\left[\tau_{t-}, \tau_{t}\right]$, and on $\left[0, \tau_{0}\right]$. In this event the process $T X=\left(X_{\tau_{t}}, \mathscr{F}_{\tau_{t}}\right)$ will be continuous if $X$ was continuous. For details and further references see the paper by Dambis [3]. 
1. The exit characteristics. Throughout this section we will assume given a process described by a measure $\boldsymbol{P}$ on path space, called the $\boldsymbol{P}$-process, and a family of strong Markov processes described by measures $\hat{\boldsymbol{P}}^{x}(x \in \bar{E})$ on the same space, such that $x \rightarrow \hat{\boldsymbol{P}}^{x}(\Lambda)$ is $\overline{\mathscr{B}}$-measurable for $\Lambda \in \mathscr{F}$, and $\hat{\boldsymbol{P}}^{x}\left[X_{0}=x\right]=1$. For such a process, which we shall refer to as the $\hat{\boldsymbol{P}}^{\cdot}$-process, the strong Markov property can be expressed as follows. For $B \in \overline{\mathscr{B}}, S$ and $T$ stopping times,

$$
\boldsymbol{E}^{x}\left[I_{B} \circ X_{T} \circ \theta_{S} \mid \mathscr{F}_{S}\right]=\hat{E}^{x}\left[I_{B} \circ X_{T}\right] \quad \hat{\boldsymbol{P}}^{x} \text {-a.s. }
$$

We write $\mu$ for the initial distribution of the $\boldsymbol{P}$-process.

We say that the $\boldsymbol{P}$-process has the same exit characteristics as the $\hat{\boldsymbol{P}}^{\cdot}$-process if the following conditions ( $\mathrm{H} 1$ and $\mathrm{H} 2$ ) hold for any $T=T_{\bar{G}^{c}}$, where $G$ is open with compact closure $\bar{G}$ in $E\left(\bar{G}^{c}=\bar{E} \mid \bar{G}\right)$, stopping time $R$, and $B \in \overline{\mathscr{B}}$.

H1. $\boldsymbol{E}\left[\boldsymbol{T} \circ \theta_{R} \mid \mathscr{F}_{R}\right]=\mathscr{E}^{X_{R}}[T] \boldsymbol{P}$-a.s.

H2. $\boldsymbol{E}\left[I_{B} \circ X_{T} \circ \theta_{R} \mid \mathscr{F}_{R}\right]=\boldsymbol{E}^{X_{R}}\left[I_{B} \circ X_{T}\right] \boldsymbol{P}$-a.s.

The main result of this section will be that under mild conditions on the $\hat{\boldsymbol{P}}$-process, this determines the $\boldsymbol{P}$-process.

We first extend properties $\mathrm{H} 1$ and $\mathrm{H} 2$ to more general stopping times, under the following hypothesis on the $\hat{\boldsymbol{P}}^{-}$-process.

F1. For each $x \in E$ there is a neighborhood $G_{x}$ with compact closure such that $\hat{E}^{y}\left[T_{\bar{G}_{x}^{c}}\right] \leqq M_{x}<\infty$ for every $y \in G_{x}$ (such sets are called exit sets in [2]).

This condition is satisfied, for instance, by any Feller process (cf. Dynkin [8, p. 139]).

Cover $E$ with a countable, locally finite collection of these exit sets, say $\left(G_{i}\right)_{i \in Z^{+}}$. Let $N(x)=\min \left\{n: x \in G_{n}\right\}$, and define $T=T_{N\left(X_{0}\right)}=T_{\bar{G}_{N\left(X_{0}\right)}}$. Let $O_{i}=G_{i} \mid \bigcup_{j<i} G_{j}$; the collection $\left(O_{\mathfrak{i}}\right)_{i \in \boldsymbol{Z}^{+}}$then forms a Borel partition of $E$ (note that $O_{i}=\{x: N(x)=i\}$ ).

LEMmA 1.1. Let $f \in C$ (the continuous bounded functions on $E$ extended to equal 0 at $\Delta), \varepsilon>0$, and $S=\inf \left\{t>0:\left|f \circ X_{t}-f \circ X_{0}\right|>\varepsilon\right\}$. The stopping time $S \wedge T$ (T defined above) then satisfies $\mathrm{H} 1$ and $\mathrm{H} 2$.

Proof. Let $A_{n k}=\left\{k 2^{-n} \leqq f<(k+1) 2^{-n}\right\}, A_{n k}^{\prime}=\left\{f>(k+1) 2^{-n}+\varepsilon\right\} \cup\left\{f<k 2^{-n}-\varepsilon\right\}$. For fixed $n$, the collection $\left\{A_{n k}\right\}_{k \in \mathbf{Z}}$ forms a Borel partition of $\bar{E}$. Let $S^{(n)}=\min \left(T_{A_{n k}}, T\right)$ if $X_{0} \in A_{n k}$. For $R$ any stopping time, $\Lambda \in \mathscr{F}_{R}, \Lambda_{i}=\Lambda \cap\left\{X_{R} \in O_{i}\right\}$, we have

$$
\begin{aligned}
E\left[S^{(n)} \circ \theta_{R} ; \Lambda_{i} \cap\left\{X_{R} \in A_{n k}\right\}\right] & =E\left[\left(T \wedge T_{A^{\prime} k}\right) \circ \theta_{R} ; \Lambda_{i} \cap\left\{X_{R} \in A_{n k}\right\}\right] \\
& =E\left[\hat{E}^{X_{R}}\left[T \wedge T_{A_{n k}^{\prime}}\right] ; \Lambda_{i} \cap\left\{X_{R} \in A_{n k}\right\}\right] \\
& =\boldsymbol{E}\left[\boldsymbol{E}^{X_{R}}\left[S^{(n)}\right] ; \Lambda_{i} \cap\left\{X_{R} \in A_{n k}\right\}\right] .
\end{aligned}
$$

Summing over $k$, we obtain

$$
\boldsymbol{E}\left[\boldsymbol{S}^{(n)} \circ \theta_{R} ; \Lambda_{i}\right]=\boldsymbol{E}\left[\hat{E}^{X_{R}}\left[S^{(n)}\right] ; \Lambda_{i}\right] .
$$

Observe that $E^{X_{R}}[T]$ is bounded for $X_{R} \in \Lambda_{i}$, so that as $n \nearrow \infty$, the dominated convergence theorem will apply. It is easily checked that $S^{(n)} \searrow S \wedge T$, so we have

$$
E\left[(S \wedge T) \circ \theta_{R} ; \Lambda_{i}\right]=E\left[E^{x_{R}}[S \wedge T] ; \Lambda_{i}\right] .
$$

Summing over $i$ establishes that $\mathrm{H} 1$ holds for $S \wedge T$. The proof for $\mathrm{H} 2$ is similar, relying on the right continuity of $t \rightarrow f \circ X_{t}$. 
Given $T, f, \varepsilon, S$ as in Lemma 1.1, we define $S_{0}=0, S_{1}=S \wedge T, S_{n+1}=S_{n}+S_{1} \circ \theta_{S_{n}}$. Clearly $S_{n}$ increases to a stopping time bounded by $T$ as $n \nearrow \infty$. Since $f \circ X_{S_{n}}$ then must converge as $n \rightarrow \infty$, we see that $S_{n}=T$ from some $n$ on, as otherwise we would have for all $n$

$$
\left|f \circ X_{S_{n+1}}-f \circ X_{S_{n}}\right|=\left|f \circ X_{S_{1}}-f \circ X_{0}\right| \circ \theta_{S_{n}} \geqq \varepsilon>0,
$$

a contradiction. We make use of this observation in the following lemma.

LEMma 1.2. For $f \in \boldsymbol{B}$ (the bounded Borel measurable functions on $E$, extended to 0 at $\Delta$,) $T$ and $R$ as above, we have

$$
\boldsymbol{E}\left[\theta_{R} \int_{0}^{T} f \circ X_{u} d u ; \Lambda\right]=E\left[E^{X_{R}}\left[\int_{0}^{T} f \circ X_{u} d u\right] ; \Lambda\right]
$$

for every $\Lambda \in \mathscr{F}_{R}$ (we use the notation $\theta_{T} F(w)=F\left(\theta_{T} w\right)$ for functions $F$ defined on $\Omega$ ).

Proof. We first assume $f \in C$, and then obtain the general case by standard approximation arguments. Let $\varepsilon>0$, and define the sequence of stopping times $\left(S_{n}\right)$ as above. For $\Lambda \in \mathscr{F}_{R}, \Lambda_{i}=\Lambda \cap\left\{X_{R} \in O_{i}\right\}$, we then have

$$
\begin{aligned}
\boldsymbol{E}\left[\theta_{R} \int_{0}^{T} f \circ X_{u} d u ; \Lambda_{i}\right]= & \boldsymbol{E}\left[\sum_{n \geqq 0} \theta_{R} \int_{S_{n}}^{S_{n+1}} f \circ X_{u} d u ; \Lambda_{i}\right] \\
= & \sum_{n \geqq 0} \boldsymbol{E}\left[f \circ X_{S_{n}} \circ \theta_{R}\left(S_{n+1}-S_{n}\right) \circ \theta_{R} ; \Lambda_{i}\right] \\
& +\sum_{n \geqq 0} \boldsymbol{E}\left[\theta_{R} \int_{S_{n}}^{S_{n+1}}\left(f \circ X_{u}-f \circ X_{S_{n}}\right) d u ; \Lambda_{i}\right] .
\end{aligned}
$$

Now the second term on the right is bounded in absolute value by

$$
\varepsilon \sum_{n \geqq 0} \boldsymbol{E}\left[\left(S_{n+1}-S_{n}\right) \circ \theta_{R} ; \Lambda_{i}\right]=\varepsilon \sum_{n \geqq 0} \boldsymbol{E}\left[\hat{E}^{X_{R}}\left[\hat{E}^{X_{S_{n}}}\left[S_{1}\right]\right] ; \Lambda_{i}\right]=\varepsilon \boldsymbol{E}\left[\hat{E}^{X_{R}}[T] ; \Lambda_{i}\right]
$$

On the other hand,

$$
\sum_{n \geqq 0} E\left[f \circ X_{S_{n}} \circ \theta_{R}\left(S_{n+1}-S_{n}\right) \circ \theta_{R} ; \Lambda_{i}\right]=\sum_{n \geqq 0} E\left[E^{x_{R}}\left[f \circ X_{S_{n}}\left(S_{n+1}-S_{n}\right)\right] ; \Lambda_{i}\right] .
$$

Similarly,

$$
E^{x_{R}}\left[\int_{0}^{T} f \circ X_{u} d u\right]=\sum_{n \geqq 0} E^{X_{R}}\left[f \circ X_{S_{n}}\left(S_{n+1}-S_{n}\right)\right]+\eta_{\varepsilon} E^{X_{R}}[T]
$$

where $0 \leqq \eta_{\varepsilon} \leqq \varepsilon$. Since $E\left[E^{x_{R}}[T] ; \Lambda_{i}\right] \leqq M_{i}$, and $\varepsilon>0$ is arbitrary, we obtain, letting $\varepsilon \searrow 0$, and then summing over $i$,

$$
\boldsymbol{E}\left[\theta_{R} \int_{0}^{T} f \circ X_{u} d u ; \Lambda\right]=E\left[\hat{E}^{X_{R}}\left[\int_{0}^{T} f \circ X_{u} d u\right] ; \Lambda\right]
$$

Lemma 1.3. For $f \in \boldsymbol{B}, R$ a stopping time, $\Lambda \in \mathscr{F}_{R}$, and $n \geqq 0$ we have

$$
E\left[\theta_{R} \int_{0}^{T} u^{n} f \circ X_{u} d u ; \Lambda\right]=E\left[E^{X_{R}}\left[\int_{0}^{T} u^{n} f \circ X_{u} d u\right] ; \Lambda\right]
$$


Proof. Again let $\Lambda_{i}=\Lambda \cap\left\{X_{R} \in O_{i}\right\}$, and assume $f \in C$. We define

$$
G(u, w)=\int_{u}^{T(w)} f \circ X_{t}(w) d t,
$$

writing $G(u)=G(u, \cdot)$ when no ambiguity arises. It is clear that $D G(u)=-f \circ X_{u}$, and that $\theta_{u} G(0)=G(u)$ on $\{u<T\}$. Integration by parts thus gives

$$
\int_{0}^{T} u^{k+1} f \circ X_{u} d u=(k+1) \int_{0}^{T} u^{k} G(u) d u .
$$

We then have, assuming that the result holds for $n=k$,

$$
\begin{aligned}
\boldsymbol{E}\left[\theta_{R} \int_{0}^{T} u^{k+1} f \circ X_{u} d u ; \Lambda_{i}\right] & =\boldsymbol{E}\left[(k+1) \theta_{R} \int_{0}^{T} u^{k} G(u) d u ; \Lambda_{i}\right] \\
& =(k+1) E\left[\int_{0}^{\infty} u^{k} \theta_{R+u} G(0) I_{\left\{T \circ \theta_{R}>u\right\}} d u ; \Lambda_{i}\right] \\
& =(k+1) \int_{0}^{\infty} u^{k} E\left[\hat{E}^{X_{R+u}}[G(0)] ;\left\{T \circ \theta_{R}>u\right\} \cap \Lambda_{i}\right] d u \\
& =(k+1) E\left[\theta_{R} \int_{0}^{T} u^{k} E^{x_{u}}[G(0)] d u ; \Lambda_{i}\right] \\
& =(k+1) E\left[\hat{E}^{x_{R}}\left[\int_{0}^{T} u^{k} \hat{E}^{x_{u}}[G(0)] d u\right] ; \Lambda_{i}\right] \\
& =E\left[E^{x_{R}}\left[\int_{0}^{T} u^{k+1} f \circ X_{u} d u\right] ; \Lambda_{i}\right]
\end{aligned}
$$

(the last equality following from a reversal of the preceding steps). The desired result for $n=k+1$ follows upon summing over $i$ (the liberal use of Fubini's theorem in the above argument is easily justified by the fact that $E^{x_{u}}[|G(0)|] \leqq M_{i}\|f\|$ on $\left\{X_{u} \in O_{i}\right\}$ ). The extension to $f \in \boldsymbol{B}$ is routine and since the case $n=0$ is just Lemma 1.2 , a use of mathematical induction finishes the job.

LemmA 1.4. For $f \in B, \lambda \geqq 0$, and $\Lambda \in \mathscr{F}_{R}-R$ a stopping time-we have

$$
\boldsymbol{E}\left[\theta_{R} \int_{0}^{T} e^{-\lambda t} f \circ X_{t} d t ; \Lambda\right]=\boldsymbol{E}\left[\hat{E}^{X_{R}}\left[\int_{0}^{T} e^{-\lambda t} f \circ X_{t} d t\right] ; \Lambda\right] .
$$

Proof. Observe that $e^{-\lambda t}=\sum_{n \geqq 0}\left((-\lambda t)^{n} / n !\right)$, a series which converges uniformly for $\lambda \leqq T<\infty$, so that

$$
\begin{aligned}
\boldsymbol{E}\left[\theta_{R} \int_{0}^{T} e^{-\lambda t} f \circ X_{t} d t ; \Lambda\right] & =E\left[\theta_{R} \int_{0}^{T} \sum_{n \geqq 0}\left((-\lambda t)^{n} / n !\right) f \circ X_{t} d t ; \Lambda\right] \\
& =\sum_{n \geqq 0}\left((-\lambda)^{n} / n !\right) E\left[E^{x_{R}}\left[\int_{0}^{T} t^{n} f \circ X_{t} d t\right] ; \Lambda\right] \\
& =E\left[E^{X_{R}}\left[\int_{0}^{T} e^{-\lambda t} f \circ X_{t} d t\right] ; \Lambda\right] \text { as desired. }
\end{aligned}
$$


So far, the only restriction we have made on the $\hat{\boldsymbol{P}}$-process is that it satisfy F1, and the only restriction on the $\boldsymbol{P}$-process is that it have the same exit characteristics as the $\hat{\boldsymbol{P}}$-process. We can now establish our main result, which is that under these conditions the $\boldsymbol{P}$-process behaves like the $\hat{\boldsymbol{P}}$-process up to time $\boldsymbol{T}$.

THEOREM 1.5. If $T$ is defined as above, if the $\hat{\boldsymbol{P}}^{\cdot}$-process satisfies $\mathrm{F} 1$, and if the $\boldsymbol{P}$-process has the same exit characteristics as the $\hat{\boldsymbol{P}}$-process, then

$$
\boldsymbol{P}[\Lambda \cap\{T>t\}]=\hat{\boldsymbol{P}}^{\mu}[\Lambda \cap\{T>t\}] \text { for } \Lambda \in \mathscr{F}_{t}, \quad t \geqq 0 .
$$

Proof. From Lemma 1.4 it follows, for $f \in C, \Lambda \in \mathscr{F}_{R}$, and

that

$$
\Lambda_{i}=\Lambda \cap\left\{X_{R} \in O_{i}\right\}
$$

$$
\boldsymbol{E}\left[\theta_{R} \int_{0}^{\infty} e^{-\lambda t} f \circ X_{t} I_{\{T>t\}} d t ; \Lambda_{i}\right]=\boldsymbol{E}\left[\hat{E}^{X_{R}}\left[\int_{0}^{\infty} e^{-\lambda t} f \circ X_{t} I_{\{T>t\}} d t\right] ; \Lambda_{i}\right],
$$

and hence that

$$
\int_{0}^{\infty} e^{-\lambda t}\left(E\left[\theta_{R} f \circ X_{t} ;\left\{T \circ \theta_{R}>t\right\} \cap \Lambda_{i}\right]-E\left[\hat{E}^{X_{R}}\left[f \circ X_{t} ; T>t ; \Lambda_{i}\right]\right) d t=0 .\right.
$$

Since $T>0$ a.s., and $t \rightarrow f \circ X_{t}$ is right continuous, it follows from Lerch's theorem for Laplace transforms (cf. [13]) that

$$
\boldsymbol{E}\left[\theta_{R} f \circ X_{t} ;\left\{T \circ \theta_{R}>t\right\} \cap \Lambda_{i}\right]=\boldsymbol{E}\left[\hat{E}^{X_{R}}\left[f \circ X_{t} ; T>t\right] ; \Lambda_{i}\right],
$$

and hence, summing over $i$, that

$$
\text { (*) } \boldsymbol{E}\left[\theta_{R} f \circ X_{t} ; T \circ \theta_{R}>t \mid \mathscr{F}_{R}\right]=\hat{E}^{X_{R}}\left[f \circ X_{t} ; T>t\right] \text {. }
$$

Taking $R=0$ and integrating against $\boldsymbol{P}$, this yields

$$
E\left[f \circ X_{i} ; T>t\right]=E\left[\hat{E}^{x_{0}}\left[f \circ X_{t} ; T>t\right]\right]=\hat{E}^{\mu}\left[f \circ X_{t} ; T>t\right] .
$$

Now let $0 \leqq t_{1}<t_{2}<\cdots<t_{n+1} \leqq t, f_{i} \in C(1 \leqq i \leqq n+1)$, and consider

$$
\text { (**) } E\left[f_{1} \circ X_{t_{1}} \cdots f_{k} \circ X_{t_{k}} ; T>t_{k}\right]=\hat{E}^{\mu}\left[f_{1} \circ X_{t_{1}} \cdots f_{k} \circ X_{t_{k}} ; T>t_{k}\right] \text {. }
$$

The equality is valid for $k=1$ by the above remarks. Supposing it is true for $k \leqq n$, let $s=t_{n+1}-t_{n}$. Then

$$
\begin{aligned}
& \boldsymbol{E}\left[f_{1} \circ X_{t_{1}} \cdots f_{n+1} \circ X_{t_{n+1}} ; t_{n+1}<T\right] \\
& =\boldsymbol{E}\left[f_{1} \circ X_{t_{1}} \cdots f_{n} \circ X_{t_{n}} f_{n+1} \circ X_{s} \circ \theta_{t_{n}} ; t_{n}+s<T\right] \\
& =\boldsymbol{E}\left[f_{n+1} \circ X_{s} \circ \theta_{t_{n}} f_{1} \circ X_{t_{1}} \cdots f_{n} \circ X_{t_{n}} ; t_{n}<T ; s<T \circ \theta_{t_{n}}\right] \\
& =\boldsymbol{E}\left[\hat{E}^{x_{t_{n}}}\left[f_{n+1} \circ X_{s} ; T>s\right] f_{1} \circ X_{t_{1}} \cdots f_{n} \circ X_{t_{n}} ; T>t_{n}\right]
\end{aligned}
$$

(by (*) above)

$$
=\hat{\boldsymbol{E}}^{\mu}\left[\hat{\boldsymbol{E}}^{\mathrm{x}_{t_{n}}}\left[f_{n+1} \circ X_{s} ; T>s\right] f_{1} \circ X_{t_{1}} \cdots f_{n} \circ X_{t_{n}} ; T>t_{n}\right]
$$

(by the induction hypothesis)

$$
=\hat{E}^{\mu}\left[f_{1} \circ X_{t_{1}} \cdots f_{n+1} \circ X_{t_{n+1}} ; T>t_{n+1}\right] \text {, as desired. }
$$


By induction (**) holds for all values of $k$ ( $n$ was arbitrary), and since functions of the form $f_{1} \circ X_{t_{1}} \cdots f_{k} \circ X_{t_{k}} I_{E} \circ X_{t}\left(0 \leqq t_{1}<\cdots<t_{k} \leqq t\right)$ generate any $\mathscr{F}_{t}$-measurable function, we are done.

In this paper we are concerned only with minimal processes, and the question is to what extent does Theorem 1.5 enable us to characterize such processes. In the case where $E$ is compact and itself an exit set, Theorem 1.5 does the job itself, since then $T=\sigma$. A completely general answer is not known, but if the $\hat{\boldsymbol{P}}$-process satisfies condition F2 below, and if $E$ is not compact, we can establish the desired characterization in terms of exit characteristics.

F2. For each $x \in E$, there is a sequence of exit sets $\left(G_{x}^{(n)}\right)$ as in $F 1$, such that $G_{x}^{(n)} \nearrow E$ as $n \nearrow \infty$ (i.e., the complements shrink down to $\{\Delta\}$ ).

This condition, though more restrictive than $F 1$, is still satisfied by a wide class of processes, as the following argument shows.

Proposition 1.6. If $\left(P_{t}\right)$ is the semigroup of a Markov process described by measures $\boldsymbol{P}^{x}(x \in \bar{E})$, and satisfies

(i) $P_{t}: C_{00} \rightarrow C\left(C_{00}\right.$ is the space of continuous functions on $E$ with compact support);

(ii) For any compact $K \subset E, P^{x}\left[T_{K^{c}}<\infty\right]>0$ for all $x \in E$;

then the function $x \rightarrow E^{x}\left[T_{K^{c}}\right]$ is bounded for any compact $K \subset E$.

Proof. We show first that for every $x \in K$ there is a neighborhood $N_{x}$ of $x$, and numbers $t_{x}>0, \varepsilon_{x}>0$, such that $\varepsilon_{x}<P^{y}\left[X_{t_{x}} \in K^{c}\right] \leqq P^{y}\left[T_{K^{c}} \leqq t_{x}\right]$ for all $y \in N_{x}$. To see this, take $K^{\prime}$ compact in $E$ such that $K$ is contained in the interior of $K^{\prime}$. Then $P^{x}\left[T_{K^{\prime c}}<\infty\right]>0$, and hence there exist $\varepsilon_{x}>0, t_{x}>0$ with

$$
P^{x}\left[X_{t_{x}} \in K^{\prime}\right]<1-\varepsilon_{x} \text {. }
$$

Letting $g \in C_{00}$ be such that $g=0$ on $K^{\prime c}, g=1$ on $K$, and $0 \leqq g \leqq 1$, we have $P_{t_{x}} g \in C$ from (i), so that $N_{x}=\left\{P_{t_{x}} g<1-\varepsilon_{x}\right\}$ is open. But clearly

$$
P_{t_{x}} g \cdot(y) \geqq P^{y}\left[X_{t_{x}} \in K\right],
$$

so $x \in N_{x}$ and $P^{y}\left[X_{t_{x}} \in K\right]<1-\varepsilon_{x}$ for all $y \in N_{x}$.

It is now routine, by covering $K$ with finitely many $N_{x}$, and letting $\delta=\min \varepsilon_{x_{i}}>0$, and $\tau=\max t_{x_{i}}$, that $P^{y}\left[T_{K^{c}} \leqq \tau\right] \geqq \delta$ for all $y \in E$. A standard argument (cf. Dynkin [8, p. 111]) now shows that $E^{y}\left[T_{K^{c}}\right] \leqq \tau / \delta$ for all $y \in K$, and since $E^{y}\left[T_{K^{c}}\right]=0$ for $y \in K^{c}$, we are done.

THEOREM 1.7. We consider only minimal processes, and $E$ is assumed to be noncompact. If the $\hat{\boldsymbol{P}}^{\boldsymbol{\theta}}$-process satisfies $\mathrm{F} 2$, and if the $\boldsymbol{P}$-process has the same exit characteristics as the $\hat{\boldsymbol{P}}_{\text {- }}$-process, then $\boldsymbol{P}=\hat{\boldsymbol{P}}^{\mu}$.

Proof. Define $T_{n}$ going with $\left(G_{x}^{(n)}\right), x \in E$, just as we defined $T$. Let $S=\lim _{n} T_{n}$. It is clear that $X_{T_{n}} \rightarrow \Delta$ as $n \nearrow \infty$ for both the $\boldsymbol{P}$ - and $\hat{\boldsymbol{P}}^{\cdot}$-processes, and from their minimal nature it follows that $S=\sigma$ a.s. for both $\boldsymbol{P}$ and $\hat{\boldsymbol{P}}^{\cdot}$. From Theorem 1.5 we have $\boldsymbol{P}=\hat{\boldsymbol{P}}^{\mu}$ for sets in $\mathscr{F}_{t} \cap\left\{T_{n}>t\right\}$ for all $n$. Letting $n \nearrow \infty$, we then have $\boldsymbol{P}=\hat{\boldsymbol{P}}^{\mu}$ on $\mathscr{F}_{t}$, for all $t$, and hence $\boldsymbol{P}=\hat{\boldsymbol{P}}^{\mu}$, as measures on $\mathscr{F}$. 
COROLlary 1.8. If the P-process has the same exit characteristics as the $\hat{\boldsymbol{P}}$-process, which we assume satisfies $\mathrm{F} 2$ and is nonterminating $\left(\sigma=\infty \hat{\boldsymbol{P}}^{-}\right.$-a.s. $)$, then $\boldsymbol{P}=\hat{\boldsymbol{P}}^{\mu}$.

Proof. The only point to check is that the sequence $T_{n} \nearrow \infty$ for the $\boldsymbol{P}$-process, which is immediate from Lemma 1.4:E[ $\left[\int_{0}^{T_{n}} e^{-\lambda t} d t\right]=E^{\mu}\left[\int_{0}^{T_{n}} e^{-\lambda t} d t\right]$, so that $\boldsymbol{E}\left[e^{-\lambda T_{n}}\right]=\hat{E}^{\mu}\left[e^{-\lambda T_{n}}\right] \searrow 0$, and hence $T_{n} \nearrow \infty \boldsymbol{P}$-a.s.

In $\S 4$ we will point out some possible extensions to nonminimal processes.

2. The continuous path case; Levy's theorem. In the case where the processes considered in $\S 1$ can be taken to have continuous paths, i.e., when $\boldsymbol{P}$ and $\hat{\boldsymbol{P}}^{x}(x \in \bar{E})$ can be taken as supported by the space of continuous paths, we can weaken the requirements for having the same exit characteristics, as follows.

Proposition 2.1. If $\boldsymbol{P}, \boldsymbol{P}^{x}(x \in \bar{E})$ are assumed to have support in the space of continuous paths, and if $\mathrm{H} 1$ and $\mathrm{H} 2$ are only assumed to hold for sets $G$ having compact closure in $E$ and belonging to a basis $\mathscr{S}$ for the topology of $E$, then $\mathrm{H} 1$ and $\mathrm{H} 2$ hold for all open sets with compact closure in $E$.

Proof. Let $G$ be open with compact closure in $E$. For $x \in E$, take $O_{x}, O_{x}^{\prime} \in \mathscr{S}$ such that $x \in O_{x} \subset \bar{O}_{x} \subset O_{x}^{\prime} \subset \bar{O}_{x}^{\prime} \subset G$. Cover $G$ with a countable locally finite collection of the sets $O_{x}$, say $\left(O_{n}\right)_{n \geqq 0}$, and form a Borel partition $\left(A_{n}\right)_{n \geqq 0}$ with $A_{n}=O_{n} \mid \bigcup_{j<n} O_{j}$. If $X_{0} \in A_{n}$, let $T=T_{\bar{O}_{n}^{\prime c}}$; if $X_{0} \in G^{c}$, let $T=0$. It is immediate that $T$ satisfies $\mathrm{H} 1$ and $\mathrm{H} 2$, as do the times $T_{n}$ constructed by letting $T_{0}=0, T_{1}=T, \ldots$, $T_{n+1}=T_{n}+T \circ \theta_{T_{n}}$. Then $T_{n} \nearrow R \leqq T_{G^{c}}$, and in fact $R=T_{G^{c}}$. Indeed, suppose $X_{R} \in G$; then $X_{R} \in A_{k}$ for some $k$. There is a neighborhood $O^{\prime}$ of $X_{R}$ which hits only finitely many $O_{i}$; take the $O_{i}^{\prime}$ associated with these, which we can assume contain $X_{R}$ (if not, just take a smaller $O^{\prime}$ to begin with). Their intersection, intersected with $O^{\prime}$, will give a neighborhood $O$ of $X_{R}$ which has the property that if $O \cap A_{i} \neq \varnothing$, then $O \backslash O_{i}^{\prime}=\varnothing$. But $X_{T_{n}}$ must lie in $O$ from some $N$ on, and this clearly gives a contradiction. Finally, to see that $\mathrm{H} 1$ and $\mathrm{H} 2$ must hold for $T_{\bar{G}^{c}}$ also, we observe that $\bar{G}$ can be written as a countable intersection of open sets with compact closure. Together with the first part of the proof, this does the job.

THEOREM 2.2. Let $X=\left(X_{t}, \mathscr{F}_{t}\right)$ be a process with values in $\boldsymbol{R}^{n}$ such that $\left(h \circ X_{t}, \mathscr{F}_{t}\right)$ is a continuous local martingale for every spherical harmonic polynomial $h(i . e ., h$ is homogeneous and $\Delta h=0)$, and such that $\left((1 / n)\left|X_{t}\right|^{2}-t, \mathscr{F}_{t}\right)$ is also a continuous local martingale. Then $X$ is a Brownian motion in $\boldsymbol{R}^{n}$.

REMARK. We will call a process $X$ in $\boldsymbol{R}^{n}$ with $h \circ X$ a continuous local martingale, for all spherical harmonic polynomials $h$ in $\boldsymbol{R}^{n}$, a continuous local martingale in $\boldsymbol{R}^{n}$.

Proof. It is a well-known fact in Lie group theory that the class $\boldsymbol{H}$ of finite linear combinations of spherical harmonics in $\boldsymbol{R}^{\boldsymbol{n}}$ is such that its restriction to the surface of any sphere about the origin is uniformly dense in the continuous functions there. 
It follows immediately from the fact that any harmonic polynomial can be written as the sum of a finite number of homogeneous harmonic polynomials that the result also holds for spheres about any point.

We write $B_{r}$ for the closed ball of radius $r$ about $O$ in $R^{n}$ (it will be clear that the result is independent of the choice of center). We take a representation of $X$ on continuous path space with measure $\boldsymbol{P}$. Fix the spherical harmonic $h$ for the moment, and suppose that $S_{n} \nearrow \infty$ is a sequence of a.s. finite stopping times such that $\left(h \circ X_{S_{n} \wedge t}, \mathscr{F}_{t}\right)$ is a continuous martingale for all $n>0$. We can clearly assume that $\left(\mathscr{F}_{t}\right)$ is a right continuous family of $\sigma$-fields, without loss of generality. We apply Doob's optional sampling theorem (cf. Meyer [12, TVI.13 and Remark VI.14b]) to the two stopping times $R^{(m)}=R \wedge m$, and $R^{(m)}+\left(k \wedge T_{B_{r}^{c}}\right) \circ \theta_{R^{(m)}}$, where $R$ is an arbitrary stopping time. This yields

$$
\boldsymbol{E}\left[h \circ X_{k \wedge S_{n} \wedge T_{B_{r}^{c}}} \circ \theta_{R^{(m)}} ; \Lambda \cap\{R<l\}\right]=\boldsymbol{E}\left[h \circ X_{S_{n} \wedge R^{(m)}} ; \Lambda \cap\{R<l\}\right],
$$

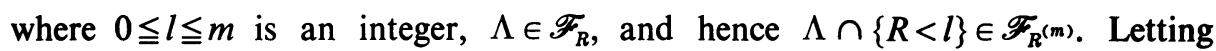
$k \nearrow \infty$, the result extends to $S_{n} \wedge T_{B_{r}^{c}}$ by the dominated convergence theorem. By Doob [6, p. 311, Theorem 3.1(ii)], the right-hand side is bounded in absolute value by $\boldsymbol{E}\left[\left|h \circ X_{R \wedge S_{n}}\right| ;\{R<l\}\right]<\infty$, so that we can again apply the dominated convergence theorem as $m \nearrow \infty$, and it only remains to let $l \nearrow \infty$ to obtain

$$
\boldsymbol{E}\left[h \circ X_{S_{n} \wedge T_{B_{r}^{c}}} \circ \theta_{R} ; \Lambda\right]=E\left[h \circ X_{R \wedge S_{n}} ; \Lambda\right] \text {. }
$$

Letting $n \nearrow \infty$, noting $X_{S \wedge T_{B_{r}^{c}}^{c}} \circ \theta_{R}$ is in $B_{r}$ when $X_{R} \in B_{r}$, we have

$$
\boldsymbol{E}\left[h \circ X_{T_{B_{r}^{c}}} \circ \theta_{R} ; \Lambda \cap\left\{X_{R} \in B_{r}\right\}\right]=E\left[h \circ X_{R} ; \Lambda \cap\left\{X_{R} \in B_{r}\right\}\right] .
$$

But on $\left\{X_{R} \in B_{r}^{c}\right\}, X_{S_{n} \wedge T_{B_{r}^{c}}} \circ \theta_{R}=X_{R}$, and so we have

$$
\boldsymbol{E}\left[h \circ X_{T_{B}^{c}} \circ \theta_{R} ; \Lambda\right]=\boldsymbol{E}\left[h \circ X_{R} ; \Lambda\right] .
$$

Given a function $f \in \boldsymbol{C}$, take a sequence $\left(h_{n}\right) \subset \boldsymbol{H}$ such that $h_{n} \rightarrow f$ uniformly on $B_{r} \backslash D_{r}\left(D_{r}=\ddot{B}_{r}\right)$. Then, since $X_{T_{B_{r}^{c}}} \in B_{r} \mid D_{r}$ for $X_{0} \in B_{r}$, we have

$$
\boldsymbol{E}\left[h_{n} \circ X_{T_{B_{r}^{c}}} \circ \theta_{R} \mid \mathscr{F}_{R}\right]=h_{n} \circ X_{R}=E^{X_{R}}\left[h_{n} \circ X_{T_{B_{r}^{c}}}\right] \quad P \text {-a.s. on }\left\{X_{R} \in B_{r}\right\} \text {, }
$$

where $\left(\hat{\boldsymbol{P}}^{x}\right)(x \in E)$ describes a Brownian motion on continuous path space. As $n \nearrow \infty$ we obtain

$$
\boldsymbol{E}\left[f \circ X_{T_{B_{r}^{c}}} \circ \theta_{R} \mid \mathscr{F}_{R}\right]=\hat{E}^{X_{R}}\left[f \circ X_{T_{B_{r}^{c}}}\right] \text { on }\left\{X_{R} \in B_{r}\right\} .
$$

Equality is obvious on $\left\{X_{R} \in B_{r}^{c}\right\}$, so we finally have

$$
\boldsymbol{E}\left[f \circ X_{T_{B_{r}^{c}}} \circ \theta_{R} \mid \mathscr{F}_{R}\right]=\hat{E}^{X_{R}}\left[f \circ X_{T_{B_{r}^{c}}}\right] \text { for } f \in \boldsymbol{C}, \boldsymbol{r}>0 .
$$

In other words, we have established $\mathrm{H} 2$ for $T_{B_{r}^{c}}$.

Now we apply optional sampling to the local martingale $\left(n^{-1}\left|X_{t}\right|^{2}-t, \mathscr{F}_{t}\right)$. As before, letting $S_{m} \nearrow \infty$ be an a.s. finite sequence of stopping times such that

$$
\left(n^{-1}\left|X_{t \wedge S_{m}}\right|^{2}-t \wedge S_{m}, \mathscr{F}_{t}\right)
$$

is a martingale for each $m$, we arrive at

$$
\boldsymbol{E}\left[\left(S_{m} \wedge T_{B_{r}^{c}}\right) \circ \theta_{R} \mid \mathscr{F}_{R}\right]=n^{-1} \boldsymbol{E}\left[\theta_{R}\left|X_{T_{B_{r}^{c} \wedge S_{m}}}\right|^{2} \mid \mathscr{F}_{R}\right]=n^{-1}\left|X_{R \wedge S_{n}}\right|^{2} .
$$


Letting $m \nearrow \infty$, we see that

$$
\begin{aligned}
& \boldsymbol{E}\left[T_{B_{r}^{c}} \circ \theta_{R} \mid \mathscr{F}_{R}\right]=n^{-1} E\left[\left|X_{T_{B_{r}^{c}}^{c}} \circ \theta_{R}\right|^{2} \mid \mathscr{F}_{R}\right]-n^{-1}\left|X_{R}\right|^{2} \\
& =n^{-1}\left(\hat{E}^{X_{R}}\left[\left|X_{T_{B_{r}^{c}}^{c}}\right|^{2}\right]-\left|X_{R}\right|^{2}\right)=\hat{E}^{X_{R}}\left[T_{B_{r}^{c}}\right] \quad P \text {-a.s., }
\end{aligned}
$$

the last equality following from the fact that $n^{-1}\left|X_{t}\right|^{2}-t$ is a martingale for Brownian motion in $\boldsymbol{R}^{n}$, which also implies that Brownian motion is nonterminating. Thus $\mathrm{H} 1$ also holds for $T_{B_{r}^{c}}$, and since it is easy to verify that $\mathrm{F} 2$ is satisfied by Brownian motion, we can apply Corollary 1.8 to obtain the desired result.

3. A characterization of continuous local martingales in $\boldsymbol{R}^{n}$. In this section we will use Theorem 2.2 to establish a characterization of all continuous local martingales in $\boldsymbol{R}^{n}$. The method is adapted from that used by Dambis [3] for continuous martingales on the line. We sketch several concepts from that paper, to which the reader is referred for details and further references.

Let $X=\left(X_{t}, \mathscr{G}_{t}\right)$ be a continuous process in $R_{n}$. Then $\lambda_{t}=t+\sup _{0 \leqq s}\left|X_{s}-X_{0}\right|$ defines a continuous, strictly increasing process $\Lambda=\left(\lambda_{t}, \mathscr{G}_{t}\right)$, and $\Theta=\Lambda^{-1}=\left(\mathscr{G}_{\theta_{t}}, \theta_{t}\right)$ is called the brake of $X$. It is easy to check that $\Theta$ is a continuous random time change, so that $\Theta X=\left(X_{\theta_{t}}, \mathscr{G}_{\theta_{t}}\right)$ is a continuous process with $\left|X_{\theta_{t}}-X_{0}\right| \leqq t$. It follows routinely from Doob's optional sampling theorem that if $X$ is a local martingale, then $X$ will be one also.

Given processes $X=\left(X_{t}, \mathscr{G}_{t}\right)$ and $Y=\left(Y_{t}, \mathscr{G}_{t}\right)$ in $\boldsymbol{R}^{n}$, and a stopping time $\tau$ for the family $\left(\mathscr{G}_{t}\right)$, we write

where

$$
[X, \tau, Y]=\left([X, \tau, Y]_{t}, \mathscr{G}_{t}\right)
$$

$$
[X, \tau, Y]_{t}=\left\{\begin{array}{l}
X_{t}, \quad 0 \leqq t<\tau, \\
Y_{t}-Y_{\tau}+X_{\tau}, \quad t \geqq \tau .
\end{array}\right.
$$

Also let $[X, \tau] \equiv[X, \tau, 0]$, and $[\tau, Y] \equiv[0, \tau, Y]$.

The following theorem is then routine (the proof follows that of Theorem 10 of [3]).

THEOREM 3.1. The process $[X, \tau, Y]$ is right continuous, and continuous in case $X$ and $Y$ are continuous. If $X$ and $Y$ are local martingales, then $[X, \tau, Y]$ is a local martingale. If $X$ and $Y$ are square integrable local martingales, then so is $[X, \tau, Y]$, and $A\left\{|[X, \tau, Y]|^{2}\right\}=\left[A\left\{|X|^{2}\right\}, \tau, A\left\{|Y|^{2}\right\}\right]$, where $A\{Z\}$ is the increasing process associated with the real valued process $Z$ by the Meyer composition.

The following lemma is the key to our result.

Lemma 3.2. Let $X=\left(X_{t}, \mathscr{G}_{t}\right)$ be a continuous local martingale in $\boldsymbol{R}^{n}$, and let $\Theta$ be the brake of $X$. Then $Y=\Theta X$ is a square integrable local martingale, and

$$
A=A\left\{|Y|^{2}\right\},
$$

is such that $A^{-1}$ is a $Y$-continuous random time change. 
Proof. The only item which we need to check is the last one. Let

$$
\tau=\inf \left\{t>0: A_{t}>0\right\}
$$

then $\tau$ is a stopping time, and it is easy to see from Theorem 3.1 that $A\left\{\left[|Y|^{2}, \tau\right]\right\}$ $=\left[A\left\{|Y|^{2}\right\}, \tau\right]=0$, so that $\left[|Y|^{2}, \tau\right]$ is a positive continuous local martingale, equal to 0 at $t=0$, and hence identically 0 (i.e., $Y_{t}=0$ for $0 \leqq t \leqq \tau$ ). Noting that

$$
A\left\{\left[q,|Y|^{2}\right]\right\}=\left[q, A\left\{|Y|^{2}\right\}\right]
$$

for any $q \geqq 0$, we have from the continuity of $A$ that $A_{q}=A_{q+s}$ implies $Y_{q}=Y_{q+t}$, $0 \leqq t \leqq s$, for all $q, s \geqq 0$, and hence $A^{-1}$ is $Y$-continuous.

TheOREM 3.3. Let $X=\left(X_{t}, \mathscr{G}_{t}\right)$ be a continuous local martingale in $\boldsymbol{R}^{n}$; then there is a continuous random time change $T$, and a family of $\sigma$-fields $\mathscr{K}_{t} \supset \mathscr{G}_{t}$, such that $X \equiv\left(X_{t}-X_{0}, \mathscr{K}_{t}\right)=T Y$, where $Y$ is a Brownian motion in $\boldsymbol{R}^{n}$, started at 0 .

Proof. With no loss of generality, we take $X_{0}=0$. Let $A=A\left\{|\Theta X|^{2}\right\}$ as in Lemma 3.2, writing $\mathscr{G}_{\theta_{t}}=\mathscr{G}_{t}^{\prime}$, and write $X^{\prime}=\Lambda \Theta X$, where $\Lambda=A^{-1}=\left(\mathscr{G}_{\lambda_{t}}^{\prime}, \lambda_{t}\right)$. Letting $\tau=A_{\infty}$, a stopping time for the family $\left(\mathscr{G}_{\lambda_{t}}^{\prime}\right)$, we see that $A\left\{\left|X^{\prime}\right|^{2}\right\}=A\left\{|\Theta X|^{2}\right\}$ $=[t, \tau]$. We may assume that the base space of the $X^{\prime}$ process is large enough to have an independent Brownian motion defined on it, say $Y=\left(Y_{t}, \mathscr{H}_{t}\right)$, with $\mathscr{H}_{\infty}$ independent of $\mathscr{G}_{\infty}$. Let $\mathscr{M}_{t}=\mathscr{H}_{t} \vee \mathscr{G}_{\lambda_{t}}^{\prime}$. Then $\tilde{Y}=\left(Y_{t}, \mathscr{M}_{t}\right)$ and $\tilde{X}^{\prime}=\left(X_{t}^{\prime}, \mathscr{M}_{t}\right)$ are respectively a Brownian motion and a continuous local martingale. Let

$$
Z=\left(Z_{t}, \mathscr{M}_{t}\right)=\left[\tilde{X}^{\prime}, \tau, \tilde{Y}\right]
$$

then $A\left\{|Z|^{2}\right\}=\left[A\left\{\left|\tilde{X}^{\prime}\right|^{2}\right\}, \tau, A\left\{|\tilde{Y}|^{2}\right\}\right]=[[t, \tau], \tau, t]=t$. Now $Z$ is clearly a continuous local martingale and so we have, from Theorem 2.2, that $Z$ is a Brownian motion. Now let $\tau_{t}=\tau \wedge t$, and $S=\left(\mathscr{M}_{t}, \tau_{t}\right)$. It is easy to check that $S Z \supset X^{\prime}$, $A X^{\prime} \supset \Theta X$, and $\Theta-1 \Theta X=X$. (We use the notation $X \supset Y$ for processes $X=\left(X_{t}, \mathscr{F}_{t}\right)$ and $Y=\left(X_{t}, \mathscr{G}_{t}\right)$ where $\left.\mathscr{F}_{t} \supset \mathscr{G}_{t}\right)$. We observe that $T=\Theta^{-1} A S$ is a continuous random time change, and so we are done.

Corollary 3.4. If $X$ in addition satisfies the conditions

(i) $X$ is a.s. not constant on any time interval, and

(ii) $\lim \sup _{t \rightarrow \infty}\left|X_{t}\right|=\infty$ a.s.,

then there is a continuous random time change $R$ such that $R X$ is a Brownian motion (i.e., $T$ is invertible in a strong sense).

Proof. Using the notation of the above proof, condition (i) insures that $A\left\{\left|\tilde{X}^{\prime}\right|^{2}\right\}$ is a strictly increasing process, and (ii) that $\tau=A_{\infty}=\infty$. Thus $A^{-1}$ is a continuous random time change, and $Z=\tilde{X}^{\prime}$ is a Brownian motion.

COROllary 3.5. Any continuous local martingale in $\boldsymbol{R}^{n}$ has the property that on any interval it is a.s. either constant or of unbounded variation.

Proof. This follows immediately from Theorem 3.3, using Theorem 2.3 of Chapter 8 of Doob [6]. 
4. Remarks and extensions. The results in $\$ 1$ are for minimal processes, i.e., for processes which are killed upon their first explosion. In order to obtain a similar characterization for nonminimal processes, one clearly must put some conditions on the return distributions. This seems to bring in, of necessity, some sort of boundary theory. A conjecture is that for processes satisfying F2, a suitable extra condition would be as follows, where $S_{\alpha}$, for $\alpha$ an ordinal, is the time of the $\alpha$ th explosion $\left(S_{1}=\sup _{n} T_{n}\right.$, with $T_{n}$ coming from F2, $S_{\alpha+1}=S_{\alpha}+S_{1} \circ S_{\alpha}$, and if $\beta$ is a limit ordinal, $\left.S_{\beta}=\sup _{\alpha<\beta} S_{\alpha}\right)$.

H3. $E\left[I_{B} \circ X_{S_{\alpha}} \circ \theta_{R} \mid \mathscr{F}_{R}\right]=\mathscr{E}^{X_{R}}\left[I_{B} \circ X_{S_{\alpha}}\right] P$-a.s., for $B \in \mathscr{B}, R$ an arbitrary stopping time.

In a future paper we will investigate the case of countable (discrete) state space processes, where the theory of the Martin boundary is well-developed and yields useful tools.

The characterization in $\S 2$ of Brownian motion can be extended to more general diffusions in $\boldsymbol{R}^{n}$. One immediate result is the following.

THeOREM 4.1. If $X$ is a continuous local martingale in $R^{n}$, and $u \circ X-t$ is $a$ continuous local martingale, where $\frac{1}{2} \Delta u=b$ a nonnegative continuous function on $\boldsymbol{R}^{n}$, then $X=T Y$, where $Y$ is a Brownian motion in $\boldsymbol{R}^{n}$, and $T$ is the (Markov) random time change inverse to the increasing process (actually an additive functional) $B$ defined by $B(t)=\int_{0}^{t} b \circ Y_{u} d u$.

Conversely, in the case where $b$ is a polynomial, a polynomial solution $u$ always exists such that $u \circ X-t$ is a martingale, where $X=T Y$ as above.

Proof. We give only a sketch of the proof. Let $u \circ X-t$ be a continuous local martingale, $X$ a continuous local martingale in $R^{n}$, and $\frac{1}{2} \Delta u=b \geqq 0$. If $B$ and $T$ are defined as above, and $Y$ is a Brownian motion (assume $X_{0}=0$ for simplicity), then let $Z=T Y$. We show that $X=Z$. Just as in the proof of Theorem 2.2 we obtain

$$
E\left[h \circ X_{T_{B}^{c}} \circ \theta_{R} \mid \mathscr{F}_{R}\right]=E^{X_{R}}\left[h \circ X_{T_{B_{r}^{c}}^{c}}\right] \text {, }
$$

for $h \in C$, and then $E\left[T_{B_{r}^{c}} \circ \theta_{R} \mid \mathscr{F}_{R}\right]=E^{X_{R}}\left[u \circ X_{T_{B_{r}^{c}}}\right]-u \circ X_{R}=E^{X_{R}}\left[T_{B_{r}^{c}}\right]$, where $P$ represents $X$ on continuous path space, and $\hat{\boldsymbol{P}}$. represents $Z$. The last equality is an immediate consequence of Dynkin's lemma (see Dynkin [8, p. 133]) and the fact that $u$ is in the domain of the weak generator for Brownian motion. The remainder of this part of the proof proceeds as in that of Theorem 2.2.

To show the existence of an appropriate $u$ in case $b$ is a polynomial in $\boldsymbol{R}^{n}$, we make use of the fact that $b$ can be written as a finite linear combination

$$
h_{0}+r^{2} h_{1}+\cdots+r^{2 k} h_{k} \text {, }
$$

where $h_{1}$ is homogeneous harmonic, and observing that for such an $h, \Delta\left(r^{2 m} h\right)=c h$, where $c$ depends on $m$ and the order and degree of homogeneity of $h$, as well as on the dimension $n$ of the space. It is then routine to solve $\frac{1}{2} \Delta u=b$, and a straightforward computation verifies that $u \circ X-t$ is indeed a martingale, where $X=T Y$ is the time change of Brownian motion with density $b$. 
Note that the difficult part of the characterization in general is showing that an appropriate solution $u$ exists, given a nice $b$. The preceding theorem solves this problem only when $b$ is a polynomial. For very general functions $b$ it is known that there is a distribution solution (à la Schwarz) of the equation $\frac{1}{2} \Delta u=b$, but it is not yet clear under what conditions this will yield a martingale function $u \circ X-t$, where $X=T Y, T$ going with $b$ as before.

Another obvious direction in which to seek further results is the following. We have so far considered only diffusions with Brownian hitting distributions (i.e., what we have called continuous local martingales in $\boldsymbol{R}^{n}$ ). In the one-dimensional case (Arbib's and Dambis' work) this is all there are, but in higher dimensions it is known (see Ito-McKean [9]) that there are others. Here more complicated elliptic partial differential operators than $\Delta$ play the role of extended generator, and a natural class of functions to examine is the kernel of one of these operators, or some suitable dense subset thereof. Now if $X$ was a continuous process with $f \circ X$ a martingale for all functions $f$ in this class, it should be possible to show that $X$ was a time change of some canonical process with the given generator. If in addition one also had $u \circ X-t$ a martingale for a suitable $u$, it should be possible to characterize $X$ exactly. This problem is currently under investigation.

\section{BIBLIOGRAPHY}

1. Michael Arbib, Hitting and martingale characterizations of one-dimensional diffusions, Z. Wahrscheinlichkeitstheorie und Verw. Gebiete 3 (1965), 232-247.

2. R. M. Blumenthal and R. K. Getoor, Additive functionals of Markov processes in a duality, Trans. Amer. Math. Soc. 112 (1964), 131-163.

3. K. E. Dambis, On the decomposition of continuous submartingales, Teor. Verojatnost. i Primenen. 10 (1965), 438-448. (Russian).

4. G. E. Denzel, The hitting characteristics of a strong Markov process, with applications to continuous martingales in $\boldsymbol{R}^{n}$, Bull. Amer. Math. Soc. 72 (1966), 1026-1027.

5. - A characterization of Markov processes in terms of their hitting characteristics, Thesis submitted to the University of Washington, August, 1965.

6. J. L. Doob, Stochastic processes, Wiley, New York, 1953.

7. L. E. Dubins and Gideon Schwarz, On continuous martingales, Proc. Nat. Acad. Sci. U.S.A. 53 (1965), 913-916.

8. E. B. Dynkin, Markov processes, Vol. 1, Springer-Verlag, Berlin, 1965.

9. K. Ito and H. P. McKean, Jr., Diffusion processes and their sample paths, Springer-Verlag, New York, 1965.

10. Hiroshi Kunita and Shinzo Watanabe, On square integrable martingales, Privately communicated preprint, 1966.

11. Paul Levy, Processus stochastiques et mouvement brownien, Gauthier-Villars, Paris, 1948; p. 78.

12. P. A. Meyer, Probability and potentials, Blaisdell, New York, 1966.

13. O. V. Widder, The Laplace transform, Princeton Univ. Press, Princeton, N. J., 1941.

Dartmouth College, HANOVER, New HaMPShiRe 